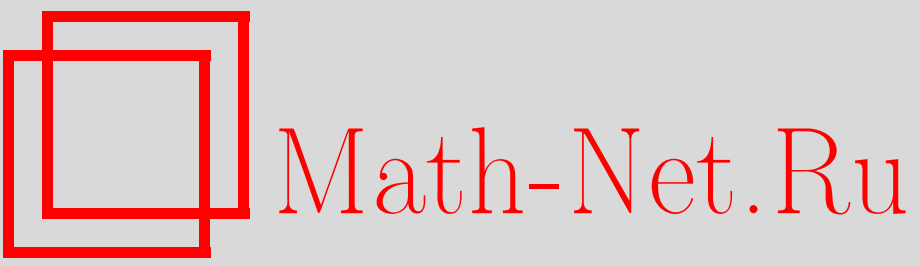

Б. Пеллони, Начальные краевые задачи для нелинейного уравнения Шредингера, ТMФ, 2000, том 122, номер 1, 128-143

DOI: https://doi.org/10.4213/tmf560

Использование Общероссийского математического портала Math-Net.Ru подразумевает, что вы прочитали и согласны с пользовательским соглашением

http://www . mathnet.ru/rus/agreement

Параметры загрузки:

IP : 3.85 .5 .30

26 апреля 2023 г., 08:31:57 
ТЕОРЕТИЧЕСКАЯ

И МАТЕМАТИЧЕСКАЯ

ФИЗИКА

Том 122, № 1

январь, 2000

(C) 2000 г.

Б. Пеллони*

\section{НАЧАЛЬНЫЕ КРАЕВЫЕ ЗАДАЧИ ДЛЯ НЕЛИНЕЙНОГО УРАВНЕНИЯ ШРЕДИНГЕРА}

Новый спектральный метод решения начальных краевых задач для линейных и интегрируемых нелинейных дифференциальных уравнений в частных производных от двух независимых переменных применен к нелинейному уравнению Шредингера и его линеаризованному варианту в области $\{x \geqslant l(t), t \geqslant 0\}$. Показано, что существует два случая: а) если $l^{\prime \prime}(t)<0$, то решение линейного и нелинейного уравнений можно получить из решения соответственно скалярной и матричной задач Римана-Гильберта $\left(\right.$ РГ) на зависящем от времени контуре; б) если $l^{\prime \prime}(t)>0$, то РГ-задача заменяется на соответственно скалярную или матричную $\bar{\partial}$-задачу в не зависящей от времени области. В обоих случаях решение выражается в виде спектрального разложения.

\section{1. ВВЕДЕНИЕ}

В работе [1] спектральный метод, предложенный в [2], применен для решения начальных краевых задач для линейных и интегрируемых нелинейных уравнений, заданных в произвольных областях вида $0 \leqslant t \leqslant T, x \geqslant l(t)$. В настояшей работе показано, как можно использовать результаты, полученные в работе [1], а именно построить решение начальной краевой задачи для линеаризованного и нелинейного уравнений Шредингера в областях $t \geqslant 0, x \geqslant l(t)$ в виде спектрального разложения.

В работе [2] исследуются различные начальные краевые задачи для линеаризованного и нелинейного уравнений Шредингера в квадранте $x \geqslant 0, t \geqslant 0$. Решение получено путем одновременного спектрального анализа обоих уравнений ассоциированной пары Лакса, а затем решения скалярной задачи Римана-Гильберта (РГ) для линейных дифференциальных уравнений в частных производных (ДУЧП) или матричной РГ-задачи для нелинейных ДУЧП. Полученное таким образом решение представлено в виде спектрального разложения. Например, для линеаризованного уравнения $i q_{t}+q_{x x}=0$ при $x \geqslant 0$ и $t \geqslant 0$ получено решение

$$
q(x, t)=\frac{1}{2 \pi} \int_{-\infty}^{\infty} e^{-i k x-i k^{2} t} \hat{q}_{0}(k) d k+\frac{1}{2 \pi} \int_{L} e^{-i k x-i k^{2} t} \nu(k) d k
$$

где $L$ - фиксированный контур в комплексной $k$-плоскости, а спектральные данные, т.е. функции $\hat{q}_{0}(k)$ и $\nu(k)$, явным образом определяются в терминах начальных и граничных

\footnotetext{
${ }^{*}$ Department of Mathematics, Imperial College, University of London, London, UK
} 
значений. С помошью этого метода получаются все глобальные соотношения, которым удовлетворяют граничные значения решения.

Для начальной краевой задачи, поставленной в произвольных областях, спектраль-

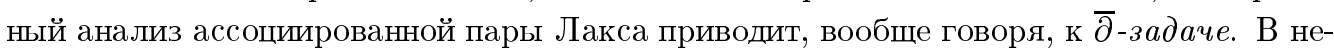
которых случаях она сводится к РГ-задаче, однако при этом контур интегрирования, используемый для представления решения, зависит от времени. Такие задачи являются скалярными, если основное ДУЧП линейно, и матрично-значными, если оно нелинейно. Полученное с помошью этого метода решение имеет вид спектрального разложения; например, для линейного уравнения $i q_{t}+q_{x x}=0, x \geqslant l(t), t \geqslant 0$, с вогнутой границей $\left(l^{\prime \prime}(t)<0\right)$, можно получить решение

$$
q(x, t)=\frac{1}{2 \pi} \int_{-\infty}^{\infty} e^{-i k x-i k^{2} t} \hat{q}_{0}(k) d k+\frac{1}{2 \pi} \int_{L_{t}} e^{-i k x-i k^{2} t} \nu(k) d k,
$$

где $L_{t}$-некоторый зависяший от времени контур, а $\hat{q}_{0}$ и $\nu(k)$ - спектральные данные, зависяшие только от начальных и граничных значений. Как и в случае одного квадранта, с помощью этого метода можно получить все глобальные соотношения, которым удовлетворяют начальные и граничные значения решения, что, таким образом, позволяет указать все корректно поставленные задачи [1].

Заметим, что в линейном случае этот метод обеспечивает как конструктивную реализацию, так и обобщение замечательного результата, известного как принцип Эренnpaйca [3]; в частности, решение данного линейного ДУЧП всегда получается в виде полного спектрального разложения (см., например, уравнения (1.1) и (1.2)).

В разделе 2 мы приводим основной результат для уравнения $i q_{t}+q_{x x}=0$. Наш метод решения может быть применен к различным начальным краевым задачам, заданным или в сложных областях, или с несепарабельными граничными условиями. Кроме того, этот метод можно делинеаризовать и использовать для решения тех же задач для полного нелинейного уравнения $i q_{t}+q_{x x}-2 \lambda|q|^{2} q=0$; этой процедуре посвящен раздел 3 .

\section{2. ЛИНЕАРИЗОВАННОЕ УРАВНЕНИЕ $i q_{t}+q_{x x}=0$}

В этом разделе мы предлагаем детальное доказательство нашего основного результата для уравнения, получаемого из нелинейного уравнения Шредингера путем отбрасывания нелинейного члена. В теореме 1 мы предполагаем, что существует единственное решение в определенном классе начальных краевых задач. В работе [1] показано, что сушествование и единственность решения можно доказать, а не постулировать, и как рассматриваемый класс начальных краевых задач соответствует классу всех корректно поставленных задач.

Теорема 1. Рассмотрим дифференциальное уравнение

$$
i q_{t}+q_{x x}=0, \quad x \geqslant l(t), \quad t \geqslant 0,
$$

где $l(t)$ - заданная гладкая вещественная ограниченная функиия такая, что $l(0)=0, a l^{\prime}(t)$ монотонна. Пусть

$$
\lim _{x \rightarrow \infty}\left|q_{x}(x, t)+q(x, t)\right|=0 \text { равномерно для } t \geqslant 0
$$

5 Теоретическая и математическая физика, т. 122, № 1, 2000 г. 


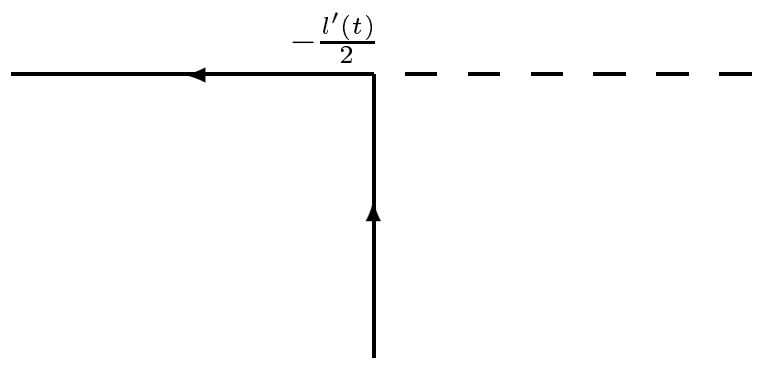

Рис.1. Контур $L(t)$ для уравнения $i q_{t}+q_{x x}=0$ при $l^{\prime \prime}(t)<0$.

$u$

$$
q(x, 0)=q_{0}(x) \in H^{2}\left(\mathbb{R}^{+}\right) .
$$

Предположим, что начальная краевая задача (2.1)-(2.3) определяется заданием граничных условий, является корректно поставленной и имеет единственное решение $q(x, t) \in \mathbb{C}$. Возможсны два случая.

Случай 1: $l^{\prime \prime}(t)<0$. Единственное решение такой корректно поставленной задачи имеет вид

$$
q(x, t)=\frac{1}{2 \pi} \int_{\mathbb{R}} e^{-i k x-i k^{2} t} \hat{q}_{0}(k) d k+\frac{1}{2 \pi} \int_{L(t)} e^{-i k x-i k^{2} t} \nu(k) d k
$$

где функиии $\hat{q}_{0}(k)$ и $\nu(k)$ определяются как

$$
\begin{aligned}
\hat{q}_{0}(k) & =\int_{0}^{\infty} e^{i k y} q_{0}(y) d y \\
\nu(k) & =\int_{0}^{\infty} e^{i\left[k^{2} s+k l(s)\right]}\left(i q_{x}(l(s), s)+\left(k+l^{\prime}(s)\right) q(l(s), s)\right) d s
\end{aligned}
$$

при этом ориентированный контур $L(t)$ является обвединением $\left\{k_{1}<-l^{\prime}(t) / 2\right.$, $\left.k_{2}=0\right\} u\left\{k_{1}=-l^{\prime}(t) / 2, \quad k_{2}<0\right\}$ (см. рис. 1). Начальные и граничные значения удовлетворяют глобальным соотношениям

$$
\begin{aligned}
& \tilde{\nu}(k)=\hat{q}_{0}(k)-e^{i k^{2} S\left(k_{1}\right)+i k l\left(S\left(k_{1}\right)\right)} \int_{0}^{\infty} e^{i k y} q\left(y-l\left(S\left(k_{1}\right)\right), S\left(k_{1}\right)\right) d y, \\
& \nu(k)=\hat{q}_{0}(k), \quad \begin{cases}k_{2}>0, & -\frac{l^{\prime}(0)}{2}<k_{1}<-\frac{l^{\prime}(\infty)}{2}, \\
k_{2}>0, & k_{1}>-\frac{l^{\prime}(\infty)}{2} .\end{cases}
\end{aligned}
$$

Здесь функиии $\hat{q}_{0}(k), \nu(k)$ даются уравнениями $(2.5),(2.6)$, а $\tilde{\nu}(k)$ определяется как

$$
\tilde{\nu}(k)=\int_{0}^{S\left(k_{1}\right)} e^{i\left[k^{2} s+k l(s)\right]} \tilde{q}(s, k) d s,
$$


$S\left(k_{1}\right)$ - обратная функция $\kappa k_{1}=-l^{\prime}(s) / 2$ :

$$
S\left(k_{1}\right)=s \quad \text { nрu } \quad k_{1}=-\frac{l^{\prime}(s)}{2}, \quad-l^{\prime}(0) \leqslant k_{1} \leqslant-l^{\prime}(\infty),
$$

$$
\tilde{q}(s, k)=i q_{x}(l(s), s)+\left(k+l^{\prime}(s)\right) q(l(s), s) .
$$

Случай 2: $l^{\prime \prime}(t)>0$. Единственное решение этой корректно поставленной задачи имеет вид

$$
\begin{aligned}
q(x, t)=\frac{1}{2 \pi} & \int_{\mathbb{R}} e^{-i k x-i k^{2} t} \hat{q}_{0}(k) d k-\frac{1}{2 \pi} \int_{-\infty}^{-\frac{l^{\prime}(\infty)}{2}} e^{-i k x-i k^{2} t} \nu(k) d k- \\
& -\frac{1}{2 \pi} \int_{-\frac{l^{\prime}(T)}{2}}^{-\frac{l^{\prime}(0)}{2}} e^{-i k x-i k^{2} t} \tilde{\nu}(k) d k+ \\
& +\frac{1}{\pi i} \iint_{D} e^{-i k x-i k^{2} t} \sigma\left(k_{1}, k_{2}\right) d k_{1} d k_{2}
\end{aligned}
$$

где функиия $\tilde{\nu}(k)$ определяется уравнением $(2.8), \sigma\left(k_{1}, k_{2}\right)$ определяется как

$$
\sigma\left(k_{1}, k_{2}\right)=-\frac{1}{l^{\prime \prime}\left(S\left(k_{1}\right)\right)} e^{i\left[k^{2} S\left(k_{1}\right)+k l\left(S\left(k_{1}\right)\right)\right]} \tilde{q}\left(S\left(k_{1}\right), k\right), \quad k \in \mathbb{D},
$$

$a \mathbb{D}$ - область вида

$$
\mathbb{D}=\left\{k:-\frac{l^{\prime}(\infty)}{2}<k_{1}<-\frac{l^{\prime}(0)}{2}, \quad k_{2}<0\right\} .
$$

Начальные и граничные значения удовлетворяют глобальному соотношению

$$
\begin{gathered}
e^{-i k^{2} S\left(k_{1}\right)-i k l\left(S\left(k_{1}\right)\right)} \nu(k)=e^{-i k^{2} S\left(k_{1}\right)-i k l\left(S\left(k_{1}\right)\right)} \hat{q}_{0}(k), \\
k_{2}>0, \quad k_{1}>-\frac{l^{\prime}(\infty)}{2},
\end{gathered}
$$

әде функиии $\hat{q}_{0}(k), \nu(k)$ и $S\left(k_{1}\right)$ определяются соответственно уравнениями $(2.5)$, (2.6) $u(2.9)$.

ДОКАЗАТЕЛЬСТвО. Приведем алгоритмическое построение, с помошью которого решение $q(x, t)$ данной начальной краевой задачи получается в виде (2.4) или (2.11). Мы покажем также, что в зависимости от природы границы это решение удовлетворяет глобальным соотношениям (2.7) или (2.13).

Уравнение (2.1) представляет собой условие совместности пары обыкновенных дифференциальных уравнений

$$
\begin{aligned}
\mu_{x}+i k \mu & =q, \\
\mu_{t}+i k^{2} \mu & =i q_{x}+k q,
\end{aligned}
$$


где $\mu=\mu(x, t, k)$. Назовем эту пару линейных уравнений парой Лакса, связанной с уравнением (2.1) [2]. Будем искать ограниченные по $k$ функции, являюшиеся решениями обоих уравнений из этой пары. После того как такие решения с конкретным асимптотическим поведением при $k \rightarrow \infty$ найдены, мы можем определить $\bar{\partial}$-задачу. Если эти решения являются также и аналитическими, то $\bar{\partial}$-задача сводится к РГ-задаче. Из решения этой $\bar{\partial}$-задачи (или РГ-задачи) можно получить решение задачи $(2.1)-(2.3)$ в форме (2.4) или (2.11).

Ограниченное для всех $k \in \mathbb{C}^{+}$решение уравнений $(2.14)$ и (2.15) имеет вид

$$
\mu_{0}(x, t, k)=-\int_{x}^{\infty} e^{-i k(x-y)} q(y, t) d y .
$$

Другое решение уравнения (2.14) имеет вид

$$
\mu(x, t, k)=e^{-i k(x-l(t))} F(t, k)+\int_{l(t)}^{x} e^{-i k(x-y)} q(y, t) d y,
$$

где $F(t, k)=\mu(l(t), t, k)$. Такая функция $\mu(x, t, k)$ является решением уравнения (2.15) тогда и только тогда, когда $\mu(l(t), t, k)$ удовлетворяет уравнениям $(2.14)$ и $(2.15)$ при $x=l(t)$, т.е. в том и только том случае, если $F$ удовлетворяет условию

$$
F_{t}+i\left(k^{2}+l^{\prime}(t) k\right) F=\tilde{q}(t, k)
$$

где $\tilde{q}(t, k)$ определяется соотношением (2.10). Поскольку входящие в $(2.17)$ экспоненты ограничены при $k \in \mathbb{C}^{-}$, то $\mu$ ограничена в $\mathbb{C}^{-}$при условии, что сушествует решение уравнения (2.18), ограниченное при $k \in \mathbb{C}^{-}$для всех $t \geqslant 0$. В решение уравнения (2.18) входит экспонента $e^{-i\left[k^{2}(t-s)+k(l(t)-l(s))\right]}$, вешественная часть показателя которой имеет вид

$$
k_{2}\left[2 k_{1}+\frac{l(t)-l(s)}{t-s}\right](t-s) .
$$

Эта экспонента ограничена в $\mathbb{C}^{-}$в том и только том случае, если ее вешественная часть отрицательна. По теореме о среднем значении из этого условия отрицательности получаем пару условий:

$$
\begin{aligned}
& 0<s<t, \quad 2 k_{1}+l^{\prime}(\tau) \geqslant 0, \quad \tau \in(s, t), \\
& t<s<\infty, \quad 2 k_{1}+l^{\prime}(\tau) \leqslant 0, \quad \tau \in(t, s) .
\end{aligned}
$$

Рассмотрим по отдельности два случая, соответствуюшие границам различной природы.

Случай 1: $l^{\prime \prime}(t)<0$. Поскольку в этом случае $l^{\prime}(t)-$ строго убывающая функция, то условие $2 k_{1}+l^{\prime}(\tau) \geqslant 0$ выполнено для всех $s<t$, если $2 k_{1}>-l^{\prime}(t)$. Аналогично, условие $2 k_{1}+l^{\prime}(\tau) \leqslant 0$ выполнено для всех $t<s$, если $2 k_{1}<-l^{\prime}(t)$. Рассмотрим в нижней полуплоскости две (зависяшие от $t$ ) области

$$
\mathbb{C}_{1}^{-}(t)=\left\{k: k_{1}>-\frac{l^{\prime}(t)}{2}\right\}, \quad \mathbb{C}_{2}^{-}(t)=\left\{k: k_{1}<-\frac{l^{\prime}(t)}{2}\right\}
$$


и получим два решения уравнений (2.14) и (2.15):

$$
\begin{aligned}
\mu_{1}(x, t, k)= & e^{-i k(x-l(t))} \int_{0}^{t} e^{-i\left[k^{2}(t-s)+k(l(t)-l(s))\right]} \tilde{q}(s, k) d s+ \\
& +\int_{l(t)}^{x} e^{-i k(x-y)} q(y, t) d y, \quad k \in \mathbb{C}_{1}^{-}, \\
\mu_{2}(x, t, k)= & -e^{-i k(x-l(t))} \int_{t}^{\infty} e^{-i\left[k^{2}(t-s)+k(l(t)-l(s))\right]} \tilde{q}(s, k) d s+ \\
& +\int_{l(t)}^{x} e^{-i k(x-y)} q(y, t) d y, \quad k \in \mathbb{C}_{2}^{-} .
\end{aligned}
$$

Определим $\hat{q}_{0}(k)$ и $\nu(k)$ с помошью соответствуюших уравнений (2.5) и (2.6). Заметим, что $\nu(k)$ ограничена при $k_{1}$ фиксированном, $k_{2}<0$ : действительно, условие $l^{\prime \prime}(t)<0$ вместе с предположением об ограниченности $l(t)$ означает, что $l^{\prime}(t)>0$; вешественная часть показателя экспоненты, входяшей в $\nu(k)$, равна $-k_{2}\left[-l^{\prime}(t) s+l(s)\right]$. Поскольку $l^{\prime}(t)>0, s>0$ и $l(s)$ ограничена, то эта экспонента ограничена при всех $k_{2}<0$.

Функции $\mu_{0}, \mu_{1}$ и $\mu_{2}$ являются аналитическими в соответствующих областях, кроме того, в области перекрытия они простым образом связаны друг с другом. Действительно, разница между двумя любыми из них должна иметь вид $e^{-i k x-i k^{2} t} \Delta(k)$. Выражение для $\Delta(k)$ можно получить, вычислив эту разницу для любых подходяших $x$ и $t$; при $x=0, t=0$ получаем

$$
\begin{array}{ll}
\mu_{0}-\mu_{1}=-e^{-i k x-i k^{2} t} \hat{q}_{0}(k), & k \in \mathbb{R}, \quad k>-\frac{l^{\prime}(t)}{2}, \\
\mu_{2}-\mu_{1}=-e^{-i k x-i k^{2} t} \nu(k), & k_{1}=-\frac{l^{\prime}(t)}{2}, \quad k_{2}<0, \\
\mu_{2}-\mu_{0}=e^{-i k x-i k^{2} t}\left[\hat{q}_{0}(k)-\nu(k)\right], & k \in \mathbb{R}, \quad k<-\frac{l^{\prime}(t)}{2} .
\end{array}
$$

Интегрирование по частям любого из представлений для $\mu$ дает

$$
\mu(x, t, k)=\frac{q(x, t)}{i k}+O\left(\frac{1}{k^{2}}\right), \quad k \rightarrow \infty, \quad k \notin L(t) \cup \mathbb{R}
$$

Уравнения (2.23) и (2.24) определяют РГ-задачу для кусочно-аналитической функции $\mu$, причем $\mu=\mu_{0}$ при $k \in \mathbb{C}^{+}, \mu=\mu_{1}$ для $\left\{k_{2}<0, k_{1}>-l^{\prime}(t) / 2\right\}$ и $\mu=\mu_{2}$ при $\left\{k_{2}<0\right.$, $\left.k_{1}<-l^{\prime}(t) / 2\right\}$ (см. рис. 2). Ее единственное решение имеет вид

$$
\mu(x, t, k)=-\frac{1}{2 \pi i}\left(\int_{\mathbb{R}} e^{-i \zeta x-i \zeta^{2} t} \frac{\hat{q}_{0}(\zeta)}{\zeta-k} d \zeta+\int_{L(t)} e^{-i \zeta x-i \zeta^{2} t} \frac{\nu(\zeta)}{\zeta-k} d \zeta\right),
$$

где $L(t)$ - контур $\left\{k_{1}<-l^{\prime}(t) / 2, \quad k_{2}=0\right\} \cup\left\{k_{1}=-l^{\prime}(t) / 2, k_{2}<0\right\}$, показанный на рис. 1. Из (2.25) и (2.24) следует решение (2.4).

Покажем теперь, что начальные и граничные значения $q(x, t)$ удовлетворяют глобальным соотношениям (2.7). Функция $\mu_{0}(x, t, k)$, определяемая уравнением $(2.16)$ при 


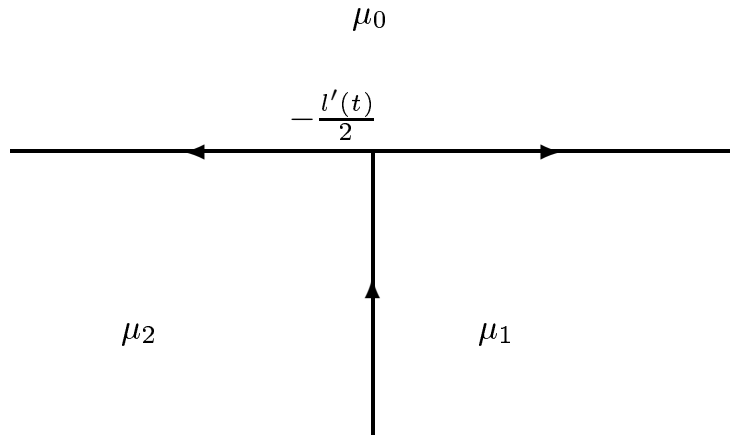

Рис.2. Области аналитичности функций $\mu_{0}, \mu_{1}, \mu_{2}$ при $l^{\prime \prime}(t)<0$.

$x=l(t)$, допускает альтернативное представление, которое можно получить из уравнения (2.18). Функция $\mu_{0}$ определяется при $k_{2}>0$, так что (2.19) принимает вид

$$
t<s, \quad 2 k_{1}+l^{\prime}(\tau)>0, \quad \tau \in(t, s) .
$$

Используя $-l^{\prime}(\tau)<-l^{\prime}(\infty)$, получаем, что неравенство (2.26) выполнено при условии, что $k_{1}>-l^{\prime}(\infty) / 2$. Таким образом, для таких значений $k_{1}$ из уравнения $(2.18)$ следует, что

$$
\mu_{0}(l(t), t, k)=-\int_{t}^{\infty} e^{-i\left[k^{2}(t-s)+k(l(t)-l(s))\right]} \tilde{q}(s, k) d s .
$$

При $-l^{\prime}(0) / 2<k_{1}<-l^{\prime}(\infty) / 2$ неравенство (2.26) выполнено только в интервале $t<$ $s<S\left(k_{1}\right)$, где $S\left(k_{1}\right)$ определяется уравнением (2.9). Действительно, при $t<\tau<s$ неравенство $2 k_{1}<-l^{\prime}(\tau)$ выполнено при условии $2 k_{1}<-l^{\prime}(s)$, которое имеет место при $s<S\left(k_{1}\right)$. Таким образом, в этом интервале из уравнения $(2.18)$ следует

$$
\begin{aligned}
\mu_{0}(l(t), t, k)=- & \int_{t}^{l\left(S\left(k_{1}\right)\right)} e^{-i\left[k^{2}(t-s)+k(l(t)-l(s))\right]} \tilde{q}(s, k) d s+ \\
& +e^{-i k^{2}\left[t-S\left(k_{1}\right)\right]-i k\left[l(t)-l\left(S\left(k_{1}\right)\right)\right]} \mu_{0}\left(l\left(S\left(k_{1}\right)\right), S\left(k_{1}\right), k\right) .
\end{aligned}
$$

Подставляя в уравнения (2.27) и (2.28) выражение для $\mu_{0}$, определяемое уравнением (2.16) при $x=l(t)$, и полагая $t=0$, получаем уравнения $(2.7)$.

Случай $2: l^{\prime \prime}(t)>0$. При $0<s<t$ в силу $-l^{\prime}(0)>-l^{\prime}(\tau)$ имеем неравенство $2 k_{1}>$ $-l^{\prime}(\tau)$, если $k_{1}>-l^{\prime}(0) / 2$. При $s<t$, аналогично, ввиду $-l^{\prime}(\infty)<-l^{\prime}(\tau)$ неравенство $2 k_{1}<-l^{\prime}(\tau)$ выполнено, если $k_{1}<-l^{\prime}(\infty) / 2$. При $-l^{\prime}(\infty) / 2<k_{1}<l^{\prime}(0) / 2$ ограниченное решение уравнения $(2.18)$ имеет вид

$$
F(t, k)=\int_{S\left(k_{1}\right)}^{t} e^{-i\left[k^{2}(t-s)+k(l(t)-l(s))\right]} \tilde{q}(s, k) d s,
$$

где при $-l^{\prime}(\infty) / 2<k_{1}<-l^{\prime}(0) / 2$ функция $S\left(k_{1}\right)$ определяется как обратная к функции $k_{1}=-l^{\prime}(s) / 2$ с помошью соотношения (2.9). Действительно, при $0<s<t$ неравенство $2 k_{1}>-l^{\prime}(\tau)$ выполнено при условии, что $2 k_{1}>-l^{\prime}(s), s<\tau<t$. Это имеет место, 
если $s>S\left(k_{1}\right), 0<s<t$, т.е. $F(t, k)$ содержит интеграл $\int_{S\left(k_{1}\right)}^{t}$ при $0<s<t$. Аналогично, $F(t, k)$ содержит интеграл $\int_{t}^{S\left(k_{1}\right)}$ при $t<s$. Таким образом, мы имеем три следуюших ограниченных решения уравнений $(2.14)$ и $(2.15): \mu_{1}(x, t, k)$, определяемое формулой $(2.21)$ при $k_{2}<0, k_{1}>-l^{\prime}(0) / 2, \mu_{2}(x, t, k)$, определяемое формулой $(2.22)$ при $k_{2}<0, k_{1}<-l^{\prime}(\infty) / 2$, и

$$
\begin{aligned}
n(x, t, k)= & e^{-i k(x-l(t))} \int_{S\left(k_{1}\right)}^{t} e^{-i\left[k^{2}(t-s)+k(l(t)-l(s))\right]} \tilde{q}(s, k) d s+ \\
& +\int_{l(t)}^{x} e^{-i k(x-y)} q(y, t) d y, \quad k_{2}<0, \quad-\frac{l^{\prime}(\infty)}{2}<k_{1}<-\frac{l^{\prime}(0)}{2} .
\end{aligned}
$$

Решение $n(x, t, k)$, определяемое формулой $(2.29)$, не является аналитическим по $k$ вследствие зависимости его от $k_{1}=(k+\bar{k}) / 2$. В результате интегрирования по частям выражений $(2.21),(2.22)$ и (2.29) получаем (2.24).

Пусть $\mu$ определяется выражениями (2.16) при $k \in \mathbb{C}^{+},(2.21)$ при $\left\{k_{2}<0, k_{1}>\right.$ $\left.-l^{\prime}(0) / 2\right\},(2.22)$ при $\left\{k_{2}<0, k_{1}<-l^{\prime}(\infty) / 2\right\}$ и $(2.29)$ при $\left\{k_{2}<0,-l^{\prime}(\infty) / 2<k_{1}<\right.$ $\left.-l^{\prime}(0) / 2\right\}$. При $k \in \mathbb{R}$ функции $\mu_{0}, \mu_{1}, \mu_{2}$ и $n$ связаны соотношениями

$$
\begin{array}{ll}
\mu_{0}-\mu_{1}=-e^{-i k x-i k^{2} t} \hat{q}_{0}(k), & k>-\frac{l^{\prime}(0)}{2}, \\
\mu_{0}-\mu_{2}=-e^{-i k x-i k^{2} t}\left[\hat{q}_{0}(k)-\nu(k)\right], & k<-\frac{l^{\prime}(\infty)}{2}, \\
n-\mu_{0}=e^{-i k x-i k^{2} t}\left[\hat{q}_{0}(k)-\tilde{\nu}(k)\right], & k \in\left(-\frac{l^{\prime}(\infty)}{2},-\frac{l^{\prime}(0)}{2}\right),
\end{array}
$$

где $\hat{q}_{0}(k), \nu(k)$ и $\tilde{\nu}(k)$ определяются уравнениями $(2.5),(2.6)$ и $(2.8)$. Значения $\mu_{1}$ и $n$ совпадают на прямой $k_{1}=-l^{\prime}(0) / 2, k_{2}<0$; аналогично, $\mu_{2}$ и $n$ совпадают на прямой $k_{1}=-l^{\prime}(\infty) / 2, k_{2}<0$. Таким образом, вдоль этих вертикальных линий не имеется скачков.

При $k \in \mathbb{C} \backslash \mathbb{D}$ функция $\mu$ является кусочно-аналитической, тогда как при $k \in \mathbb{D}$ она не является аналитической. Чтобы найти интегральное представление для $\mu$, нам потребуется кроме уравнений (2.30) вычислить $\bar{\partial}$-производную от $\mu$, т.е. $\bar{\partial} \mu=\partial \mu / \partial \bar{k}$. Из уравнения (2.29) следует

$$
\bar{\partial} n(x, t, k)=e^{-i k x-i k^{2} t} \sigma\left(k_{1}, k_{2}\right),
$$

где $\sigma$ определяется уравнением (2.12). Заметим, что $\bar{\partial}$-производная $\bar{\partial} n(x, t, k)$ хорошо определена при $k \in \mathbb{D}$. Действительно, при $k \in \mathbb{D}$ экспонента имеет вешественную часть, равную $k_{2}\left(x+2 k_{1} t\right)$. Поскольку $x \geqslant l(t)$ и $2 k_{1}>-l^{\prime}(\infty)$, имеем

$$
x+2 k_{1} t>t\left(\frac{l(t)}{t}-l^{\prime}(\infty)\right)=t\left(l^{\prime}(\tau)-l^{\prime}(\infty)\right)>0, \quad 0<\tau<t
$$




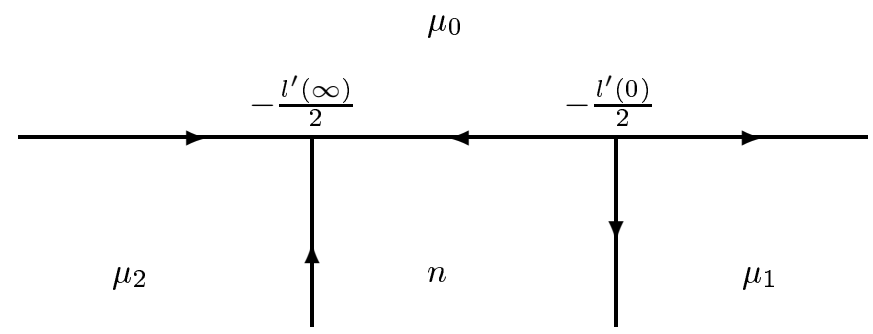

Рис. 3. Области аналитичности решений $\mu_{0}, \mu_{1}, \mu_{2}$ и область ограниченности решения $n$ для случая $l^{\prime \prime}(t)>0$.

Поскольку $k_{2}<0$, эта вешественная часть отрищательна, и экспонента является ограниченной.

Уравнения (2.30) и (2.31) вместе с уравнением (2.24) определяют $\bar{\partial}$-задачу. Из обобщенной формулы Коши [4]

$$
\begin{gathered}
\mu(x, t, k)=\frac{1}{2 \pi i} \int_{\partial D} \frac{\mu(x, t, \zeta)}{\zeta-k} d \zeta-\frac{1}{\pi} \iint_{D} \frac{\bar{\partial} \mu(x, t, \zeta)}{\zeta-k} d \xi d \eta \\
k \in D, \quad \zeta=\xi+i \eta
\end{gathered}
$$

для функции $\mu$, показанной на рис. 3 , следует, что единственное решение такой $\bar{\partial}$-задачи имеет вид

$$
\begin{aligned}
\mu= & -\frac{1}{2 \pi i} \int_{\mathbb{R}} e^{-i \zeta x-i \zeta^{2} t} \frac{\hat{q}_{0}(\zeta)}{\zeta-k} d \zeta+\frac{1}{2 \pi i} \int_{-\infty}^{a_{0}} e^{-i \zeta x-i \zeta^{2} t} \frac{\tilde{\nu}(\zeta)}{\zeta-k} d \zeta- \\
& -\frac{1}{\pi} \iint_{D} \frac{e^{-i \zeta x-i \zeta^{2} t} \sigma(\xi, \eta)}{\zeta-k} d \xi d \eta .
\end{aligned}
$$

Из последнего уравнения и формулы (2.24) получаем решение (2.11).

Покажем теперь, что начальные и граничные значения $q(x, t)$ удовлетворяют глобальному соотношению (2.13). Как и в случае 1 , рассмотрим альтернативное представление $\mu_{0}(l(t), t, k)$ в верхней полуплоскости, полученное из уравнения (2.18). Исследуя ограниченность соответствующего показателя экспоненты, можно показать, что при $k_{1}>-l^{\prime}(t) / 2$ это представление имеет вид

$$
\mu_{0}(l(t), t, k)=-\int_{t}^{\infty} e^{-i\left[k^{2}(t-s)+k(l(t)-l(s))\right]} \tilde{q}(s, k) d s .
$$

Подставляя в полученное соотношение выражение $(2.16)$, вычисленное при $x=l(t)$, и полагая $t=0$, при $k_{1}>-l^{\prime}(0) / 2$ получим $\nu(k)=\hat{q}_{0}(k)$. Отметим, что если мы умножим обе части этого уравнения на экспоненту $e^{-i k^{2} S\left(k_{1}\right)-i k l\left(S\left(k_{1}\right)\right)}$, то это соотношение 
можно будет аналитически продолжить в область $\left\{k_{1}>-l^{\prime}(\infty) / 2, k_{2}>0\right\}$. Действительно, вешественная часть соответствуюшего показателя экспоненты равна $k_{2}\left(2 k_{1}+\right.$ $\left.l^{\prime}(\tau)\right)\left(S\left(k_{1}\right)-s\right)$, где $\tau$ - некоторая точка в интервале между $S\left(k_{1}\right)$ и $s, 0<s$. Если $S\left(k_{1}\right)<\tau<s$, то этот показатель отрицателен в случае $2 k_{1}+l^{\prime}(\tau)>0$. Это всегда выполняется, поскольку $2 k_{1}=-l^{\prime}\left(S\left(k_{1}\right)\right)>-l^{\prime}(\tau)$. Аналогичные рассуждения верны для случая $s<\tau<S\left(k_{1}\right)$. Таким образом, глобальное условие (2.13) хорошо определено и сохраняется при $k_{1}>-l^{\prime}(\infty)$ и $k_{2}>0$.

ЗАмЕчАниЕ 1. Глобальные соотношения (2.7) и (2.13) необходимы, для того чтобы указать все корректно поставленные начальные краевые задачи. Строгое доказательство сушествования решения начальной краевой задачи можно получить, только если принять, что граничные значения удовлетворяют этим соотношениям. Доказательство этого факта приведено в работе [1].

ЗАмечАние 2. Решения (2.4) и (2.11) можно переписать в виде

$$
\begin{aligned}
q(x, t)= & \frac{1}{2 \pi} \int_{\mathbb{R}} e^{-i k x-i k^{2} t} \hat{q}_{0}(k) d k- \\
& -\frac{1}{2 \pi} \int_{\mathbb{R}} e^{-i k x-i k^{2} t} \int_{0}^{t} e^{i k^{2} s+i k l(s)} \tilde{q}(s, k) d s d k .
\end{aligned}
$$

Соотношение (2.34) можно получить, непосредственно решив уравнение (2.1) с помощью преобразования Фурье. Такое представление не дает спектрального разложения для $q(x, t)$, более того, из этой формулы не ясно, какие именно начальные краевые задачи являются корректно поставленными. Тем не менее из эквивалентности решений (2.4) и (2.11) с решением (2.34) немедленно следует вывод о правильности исходного уравнения.

\section{3. НЕЛИНЕЙНОЕ УРАВНЕНИЕ ШРЕДИНГЕРА}

В этом разделе мы покажем, как метод, с помощью которого решаются начальные краевые задачи для уравнения $q_{t}+i q_{x x}=0$, может быть использован для изучения аналогичных задач для нелинейного уравнения Шредингера. Как и в разделе 2 , наш анализ разбивается на два случая в зависимости от природы границы. В первом случае решение получается с помощью матричной РГ-задачи, а во втором - матричной $\bar{\partial}$-задачи. Полное доказательство можно найти в работе [1].

Имеются два важных отличия линейного случая от интегрируемого нелинейного. Первое отличие заключается в том, что в линейном случае спектральные данные явно характеризуются решением линейного интегрального уравнения Вольтерра (см. [1]), а в нелинейном случае спектральные данные можно охарактеризовать только с помощью нелинейного интегрального уравнения. Второе отличие состоит в том, что нелинейное уравнение поддерживает сушествование солитонных решений. Такие решения возникают как следствие того факта, что в нелинейном случае решение соответствуюшей РГ-задачи или $\bar{\partial}$-задачи имеет полюсы на комплексной плоскости $k$. Например, в 
случае 1 теоремы 2 функции $\mu(x, t, k)$, представляющие собой решения РГ-задачи, являются кусочно-мероморфными, а не кусочно-аналитическими.

Подчеркнем, что РГ-задача (случай 1 теоремы 2) может быть решена без предположения о малости нормы спектральных данных. Детальное доказательство разрешимости РГ-задачи, аналогичное предложенному здесь, можно найти в работе [5]. В случае $\bar{\partial}$-задачи (случай 2 теоремы 2) нам по-прежнему приходится использовать предположение о малости нормы для доказательства сушествования.

Сформулируем основной результат для двух случаев и в случае 1 покажем, как можно использовать для него доказательство теоремы 1.

ТЕОРема 2. Пусть $q(x, t) \in \mathbb{C}$ удовлетворяет нелинейному уравнению Шредингера

$$
i q_{t}+q_{x x}-2 \lambda|q|^{2} q=0, \quad x \geqslant l(t), \quad t \geqslant 0, \quad \lambda^{2}=1
$$

где $l(t)$ - заданная гладкая вещественная ограниченная функиия такая, что $l^{\prime}(t)$ строго монотонна, a $l(0)=0$. Предположим, что

$$
\lim _{x \rightarrow \infty}\left|q(x, t)+q_{x}(x, t)\right|=0 \quad \text { равномерно по } t \geqslant 0
$$

и $q(x, 0)=q_{0}(x) \in \mathcal{S}\left(\mathbb{R}^{+}\right)$. Допустим, что заданные граничные условия такови, что существует гладкое глобальное решение. Это решение удовлетворяет условию

$$
q(x, t)=2 i \lim _{k \rightarrow \infty} \mu_{12}(x, t, k)
$$

где $\mu_{12}$ обозначает әлемент $(1,2)$ комплекснозначной $(2 \times 2)$-матрицы $\mu(x, t, k)$. Возможсны два случая.

Случай 1: $l^{\prime \prime}(t)<0$. Матрица $\mu(x, t, k)$ представляет собой единственное решение РГ-задачи, поставленной следующим образом:

- определитель н равен единице;

- $\mu$ является мероморфной функцией на комплексной k-плоскости, разрезанной nо кресту

$$
L_{t}=\left\{k_{1}=-\frac{l^{\prime}(t)}{4}\right\} \cup\left\{k_{2}=0\right\} ;
$$

- $\mu$ удовлетворяет условию

$$
\mu=I+O\left(\frac{1}{k}\right), \quad k \rightarrow \infty, \quad k \notin L_{t}
$$

- на контуре $L_{t}$ функция $\mu$ удовлетворяет условиям скачка

$$
\mu^{-}(x, t, k)=\mu^{+}(x, t, k) J(\theta(x, t), k), \quad k \in L_{t},
$$

где $\theta(x, t)=2 i k x+4 i k^{2} t$, а вид $(2 \times 2)$-матрицы скачка $J$ показан на рис. 4. 


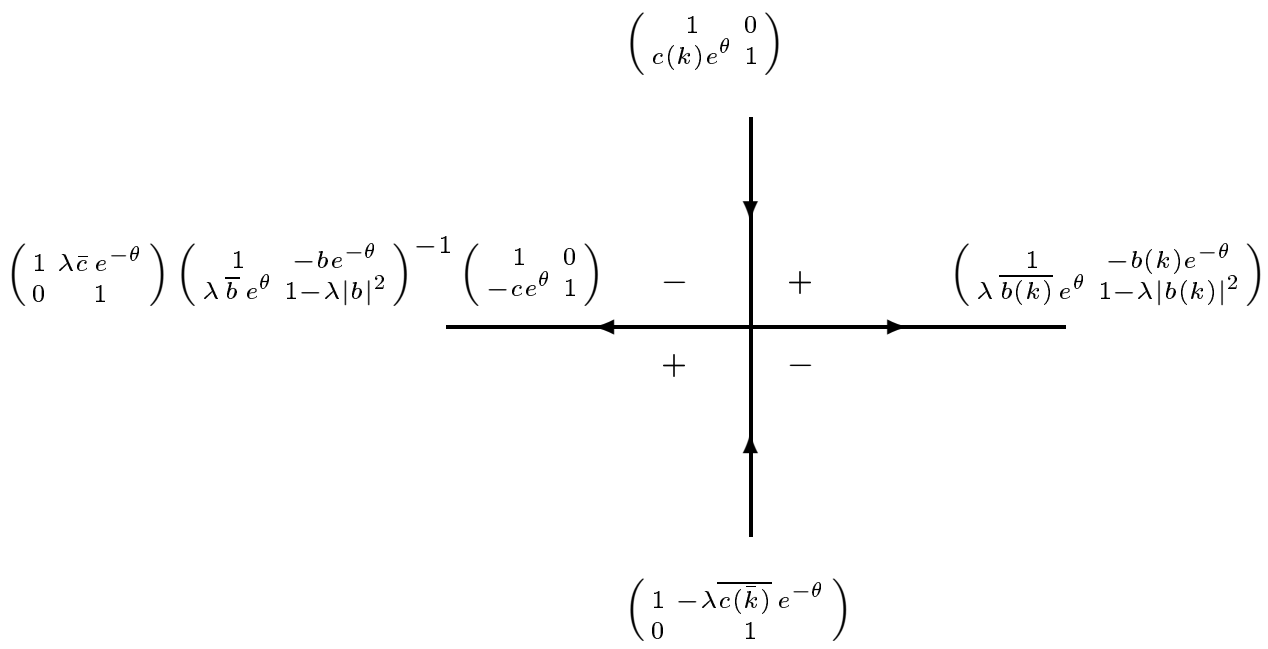

Рис. 4. Матрица скачка для РГ-задачи, связанной с начальной краевой задачей для нелинейного уравнения Шредингера при $x \geqslant l(t), t \geqslant 0$ в случае $l^{\prime \prime}(t)<0$. Горизонтальная линия соответствует $\operatorname{Re} k=0$, а вертикальная $-\operatorname{Im} k=-l^{\prime}(t) / 4$. Матрица скачка зависит от переменных $x, t$ только через функцию $\theta(x, t)=2 i k x+4 i k^{2} t$.

Скаляры $b(k)$ и $c(k)$ определяются через граничные значения $q(x, t)$ как

$$
\begin{gathered}
b(k)=\frac{m_{1}^{+}(0, k)}{m_{2}^{+}(0, \bar{k})}, \quad c(k)=\frac{\lambda \overline{\varphi_{1}^{(3)}}(0, \bar{k})}{m_{2}^{+}(0, k) d(k)}, \\
d(k)=m_{2}^{+}(0, k) \overline{\varphi_{2}^{(3)}}(0, \bar{k})-\lambda m_{1}^{+}(0, k) \overline{\varphi_{1}^{(3)}}(0, \bar{k}),
\end{gathered}
$$

причем величины, появляющиеся в выражениях (3.3) и (3.4), можно вычислить с помощьюо векторов

$$
\begin{aligned}
m^{+}(x, k) & =\left(m_{1}^{+}(x, k), m_{2}^{+}(x, k)\right)^{\tau}, \\
\varphi^{(3)}(t, k) & =\left(\varphi_{1}^{(3)}(t, k), \varphi_{2}^{(3)}(t, k)\right)^{\tau},
\end{aligned}
$$

которые являются единственными решениями обыкновенных дифференциальных уравнений

$$
\begin{gathered}
m_{x}^{+}+2 i k\left(\begin{array}{ll}
1 & 0 \\
0 & 0
\end{array}\right) m^{+}=\left(\begin{array}{cc}
0 & q_{0}(x) \\
\lambda \bar{q}_{0}(x) & 0
\end{array}\right) m^{+}, \\
\lim _{x \rightarrow \infty} m^{+}=(0,1)^{\tau}, \quad x \geqslant 0, \quad k \in \mathbb{C}^{+},
\end{gathered}
$$


$u$

$$
\begin{gathered}
\varphi_{t}^{(3)}+\left(4 i k^{2}+2 i k l^{\prime}(t)\right)\left(\begin{array}{ll}
1 & 0 \\
0 & 0
\end{array}\right) \varphi^{(3)}= \\
=\left(\begin{array}{cc}
-i \lambda|q(t)|^{2} & i q_{x}(t)+\left(2 k+l^{\prime}(t)\right) q(t) \\
-i \lambda \bar{q}_{x}(t)+i \lambda\left(2 k+l^{\prime}(t)\right) \bar{q}(t) & i \lambda|q(t)|^{2}
\end{array}\right) \varphi^{(3)}, \\
\lim _{t \rightarrow \infty} \varphi^{(3)}=(0,1)^{\tau}, \quad t \geqslant 0, \quad k_{1}>-\frac{l^{\prime}(t)}{4}, \quad k_{2}>0, \\
q(t)=q(l(t), t), \quad q_{x}(t)=q_{x}(l(t), t) .
\end{gathered}
$$

Единственнье полюсы функиии н могут появиться в нулях функиии $m_{2}^{+}(0, k)$ при $k \in \mathbb{C}_{1}(t)=\left\{k: k_{1}>-l^{\prime}(t) / 4, \quad k_{2}>0\right\}$ и в нулях функиии $d(k)$ при $k \in \mathbb{C}_{2}(t)=\{k:$ $\left.k_{1}<-l^{\prime}(t) / 4, \quad k_{2}>0\right\}$, а также для значений, комплексно-сопряженных этим нулям.

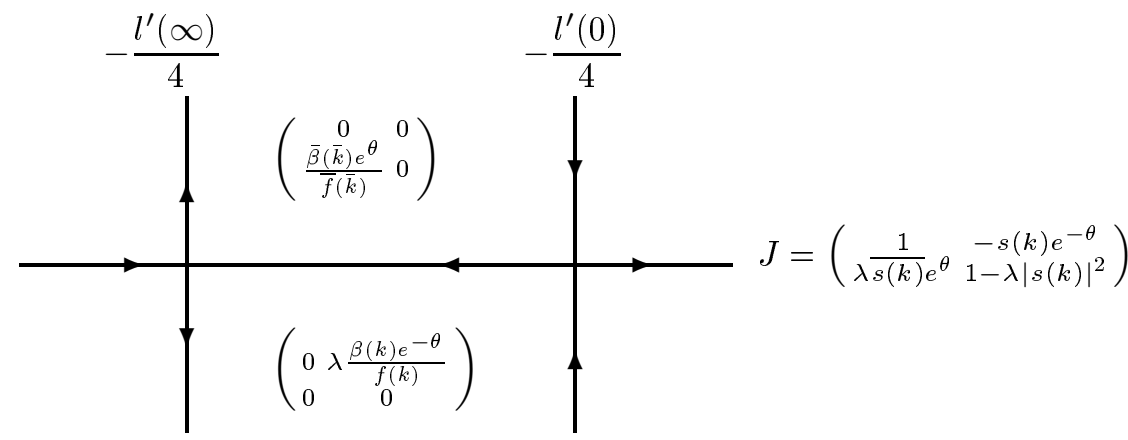

Рис. 5. Матрица скачка вдоль $\mathbb{R}$ и данные $\bar{\partial}$-задачи, связанной с нелинейным уравнением Шредингера при $l^{\prime \prime}(t)>0$. Матрица скачка зависит от переменных $x, t$ только через функцию $\theta(x, t)=2 i k x+4 i k^{2} t$.

Случай 2: $l^{\prime \prime}(t)>0$. При достаточно мальх $q(x, t)$ матрица $\mu(x, t, k)$ является

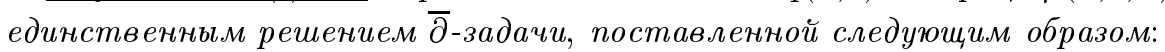

- определитель н равен единице;

- $\mu$ является функиией, ограниченной на комплексной $k$-плоскости, разрезанной вдоль вещественной оси $\mathbb{R}$, и мероморфной при $k_{1}>-l^{\prime}(0) / 4$ и при $k_{1}<$ $-l^{\prime}(\infty) / 4$

- $\mu$ удовлетворяет условию

$$
\mu=I+O\left(\frac{1}{k}\right), \quad k \rightarrow \infty, \quad \operatorname{Im} k \neq 0
$$

- вдоль $\mathbb{R}$ функиия $\mu$ удовлетворяет условиям скачка

$$
\mu^{-}(x, t, k)=\mu^{+}(x, t, k) J(\theta(x, t), k),
$$

где $\theta(x, t)=2 i k x+4 i k^{2} t$, а вид $(2 \times 2)$-матрицы скачка $J(\theta(x, t), k)$ показан на рис. 5. 
Более того, $\partial \mu / \partial \bar{k}=S(\theta(x, t), k, \bar{k}) \mu$ при $k \in D($ вид функции $S$ также показан на puc. 5).

Скаляры $s(k), \beta(k)$ и $f(k)$ определяются в терминах граничных значений решения $q(x, t)$ как

$$
\begin{aligned}
& s(k)= \begin{cases}\frac{m_{1}^{+}(0, k)}{m_{2}^{+}(0, k)}, & k>-\frac{l^{\prime}(0)}{4}, \\
\frac{\varphi_{2}^{(3)}(0, k) m_{1}^{+}(0, k)-\varphi_{2}^{(3)}(0, k) m_{2}^{+}(0, k)}{d(k)}, & k<-\frac{l^{\prime}(\infty)}{4}, \\
\frac{m_{1}^{+}(0, k) n_{2}^{(6)}(0, k)-m_{2}^{+}(0, k) n_{1}^{(6)}(0, k)}{f(k)}, & -\frac{l^{\prime}(\infty)}{4}<k<-\frac{l^{\prime}(0)}{4},\end{cases} \\
& d(k)=\varphi_{2}^{(3)}(0, k) \overline{m_{2}^{+}}(0, \bar{k})-\lambda \varphi_{1}^{(3)}(0, k) \overline{m_{1}^{+}}(0, \bar{k}), \\
& k_{1}<-\frac{l^{\prime}(\infty)}{4}, \quad k_{2}<0, \\
& f(k)=\overline{m_{2}^{+}}(0, \bar{k}) n_{2}^{(6)}(0, k)-\lambda \overline{m_{1}^{+}}(0, \bar{k}) n_{1}^{(6)}(0, k), \\
& -\frac{l^{\prime}(\infty)}{4}<k_{1}<-\frac{l^{\prime}(0)}{4}, \quad k_{2}<0, \\
& \beta(k)=-\frac{1}{2 l^{\prime \prime}\left(S\left(k_{1}\right)\right)} \frac{\left(2 k q+l^{\prime}\left(S\left(k_{1}\right)\right) q+i q_{x}\right) e^{\theta}}{\overline{\vec{m}}_{1}\left(S\left(k_{1}\right), \bar{k}\right)},
\end{aligned}
$$

где функиии $q(t)=q(l(t), t), \quad q_{x}(t)=q_{x}(l(t), t), \quad \theta(t, k)=\theta(l(t), t, k)$ вычислены при $t=S\left(k_{1}\right)$, а $S\left(k_{1}\right)$ определяется из условия

$$
S\left(k_{1}\right)=s, \quad \text { ecлu } \quad k_{1}=-\frac{l^{\prime}(s)}{4} .
$$

Другие величинь, появляющиеся в определениях (3.7)-(3.9), можно вычислить с помощью векторов

$$
\begin{aligned}
m^{+}(x, k) & =\left(m_{1}^{+}(x, k), m_{2}^{+}(x, k)\right)^{\tau}, \\
\varphi^{(3)}(t, k) & =\left(\varphi_{1}^{(3)}(t, k), \varphi_{2}^{(3)}(t, k)\right)^{\tau}, \\
n^{(6)}(t, k) & =\left(n_{1}^{(6)}(t, k), n_{2}^{(6)}(t, k)\right)^{\tau}, \\
\widetilde{m}(t, k) & =\left(\widetilde{m}_{1}(t, k), \widetilde{m}_{2}(t, k)\right)^{\tau},
\end{aligned}
$$

которые являются единственными решениями следующих уравнений: $m^{+}(x, k)$ удовлетворяет уравнению $(3.5) ; \varphi^{(3)}(t, k), n^{(6)}(t, k)$ и $\widetilde{m}(t, k)$ удовлетворяют уравнению (3.6) везде кроме $\left\{k: k_{1}<-l^{\prime}(\infty) / 4, k_{2}<0\right\},\left\{k:-l^{\prime}(\infty) / 4<k_{1}<-l^{\prime}(0) / 4\right.$, $\left.k_{2}<0\right\}$ u $\left\{k: k_{1}>-l^{\prime}(0) / 4, k_{2}>0\right\}$, соответственно. 
НАБРОСОК ДОКАЗАТЕЛЬСТВА. Нелинейное уравнение Шредингера обладает парой Лакса [6]

$$
\begin{aligned}
\mu_{x}+i k\left[\sigma_{3}, \mu\right] & =Q \mu, \\
\mu_{t}+2 i k^{2}\left[\sigma_{3}, \mu\right] & =\widetilde{Q} \mu,
\end{aligned}
$$

где $\mu(x, t, k)$ - комплексная $(2 \times 2)$-матрично-значная функция, а матрицы $Q(x, t)$ и $\widetilde{Q}(x, t)$ определяются как

$$
Q=\left(\begin{array}{cc}
0 & q \\
\lambda \bar{q} & 0
\end{array}\right), \quad \widetilde{Q}=2 k Q-i Q_{x} \sigma_{3}-i \lambda|q|^{2} \sigma_{3} .
$$

По аналогии с функциями $\mu_{0}, \mu_{1}, \mu_{2}, n$, задаваемыми в линеаризованном случае соответственно уравнениями $(2.16),(2.21),(2.22)$ и $(2.29)$, введем $(2 \times 2)$-матрицы

$$
M=\left(M^{-}, M^{+}\right), \quad \Psi=\left(\Psi^{1}, \Psi^{4}\right), \quad \Phi=\left(\Phi^{2}, \Phi^{3}\right),
$$

где

$$
\begin{aligned}
M(x, t, k)= & I+\int_{x}^{\infty} e^{-i k(x-y) \hat{\sigma}_{3}} Q(y, t) M(y, t, k) d y . \\
\Psi(x, t, k)=I & +\int_{l(t)}^{x} e^{-i k(x-y) \hat{\sigma}_{3}} Q(y, t) \Psi(y, t, k) d y+ \\
& +e^{-i k(x-l(t)) \hat{\sigma}_{3}} \int_{0}^{t} e^{-i\left[2 k^{2}(t-s)+k(l(t)-l(s))\right] \hat{\sigma}_{3}} \times \\
& \times\left[\left(\widetilde{Q}+l^{\prime}(s) Q\right) \Psi\right](l(s), s, k) d s,
\end{aligned}
$$

а вид $\Phi(x, t, k)$ подобен $(3.14)$ с заменой интеграла $\int_{0}^{t}$ на интеграл $\int_{t}^{\infty}$. Здесь $S\left(k_{1}\right)$ определяется уравнением (3.10) и мы используем обозначение

$$
e^{x \hat{\sigma}_{3}} A=e^{x \sigma_{3}} A e^{-x \sigma_{3}} .
$$

Векторы-столбцы этих матриц ограничены в областях, показанных на рис. 6. Используя эти функции, несложно обобщить построения, проведенные в разделе 2 , и определить РГ-задачу, поставленную в теореме 2 .

Например, функцию $\mu(x, t, k)$ можно записать в каноническом виде

$$
\begin{array}{lll}
\left(\frac{\Psi^{1}}{m_{2}^{+}(0, k)}, M^{+}\right), & k \in \mathbb{C}_{1}(t) ; & \left(\frac{\Phi^{2}}{d(k)}, M^{+}\right), \quad k \in \mathbb{C}_{2}(t) ; \\
\left(M^{-}, \frac{\Phi^{3}}{\bar{d}(\bar{k})}\right), \quad k \in \mathbb{C}_{3}(t) ; & \left(M^{-}, \frac{\Psi^{4}}{m_{2}^{+}(0, \bar{k})}\right), k \in \mathbb{C}_{4}(t) ;
\end{array}
$$

где $m^{+}(k)=M^{+}(x, 0, k), \varphi^{(3)}(t, k)=\Phi^{3}(l(t), t, k), d(k)$ определяется уравнением $(3.4)$, а области $\mathbb{C}_{1}, \ldots, \mathbb{C}_{4}$ показаны на рис. 6 . Такая функция $\mu$ удовлетворяет условию скачка, изображенному на рис. 4 . 


\begin{tabular}{ll|l}
$\Phi^{2}, M^{+}$ & & \multicolumn{2}{|c}{$\Psi^{1}, M^{+}$} \\
& $\mathbb{C}_{2}$ & $\mathbb{C}_{1}$ \\
\hline & $\mathbb{C}_{3}$ & $\mathbb{C}_{4}$ \\
$M^{-}, \Phi^{3}$ & & \multicolumn{1}{|c}{$M^{-}, \Psi^{4}$}
\end{tabular}

Рис. 6. Нелинейное уравнение Шредингера в случае $l^{\prime \prime}(t)<0$. Области аналитичности функций $M=\left(M^{-}, M^{+}\right), \Phi=\left(\Phi^{2}, \Phi^{3}\right), \quad \Psi=\left(\Psi^{1}, \Psi^{4}\right)$. Центр креста - точка $k=-l^{\prime}(t) / 2$.

Из уравнений (3.15) следует, что функции, определяюшие РГ-задачу, могут иметь полюсы в нулях функций $m_{2}^{+}(k)$ и $d(k)$ и в комплексно-сопряженных этим нулям точках. Можно отобразить описанную выше РГ-задачу на РГ-задачу, содержашую только аналитические функции, дополненные системой алгебраических уравнений. В этом случае анализ аналогичен тому, который детально описан в работе [5].

Благодарности. Настоящая работа является частью совместного проекта с А. С. Фокасом, которому автор очень благодарен за многочисленные существенные советы. Работа поддержана программой TMR EC (грант № ERBFMBICT961561).

\section{Список литературы}

[1] A. S. Fokas, B. Pelloni. A spectral method for linear and integrable nonlinear PDE's in arbitrary domains (unpublished); A method of solving moving boundary value problems for linear evolution equations (submitted to Phys. Rev. Lett.).

[2] A. S. Fokas. Proc. Roy. Soc. Ser. A. 1997. V. 453. P. 1411-1443.

[3] L. Ehrenpreis. Fourier Analysis in Several Complex Variables. New York: Wiley-Interscience, 1970.

[4] M. J. Ablowitz, A. S. Fokas. Introduction and Applications of Complex Variables. Cambridge: Cambridge Univ. Press, 1997.

[5] A. S. Fokas, A. Its. SIAM J. Math. Anal. 1996. V. 27. P. 738-764.

[6] В. Е. Захаров, А. Б. Шабат. ЖЭТФ. 1971. Т. 61. С. 118-134. 